\title{
Energy-Efficient and Labor-Aware Production Scheduling based on Multi-Objective Optimization
}

\author{
Xu Gong*, Toon De Pessemier, Luc Martens, Wout Joseph \\ Ghent University/imec, Technologiepark 15, 9052 Ghent, Belgium \\ xu.gong@ugent.be
}

\begin{abstract}
Manufacturing industry is a major energy consumer and greenhouse gas producer for the society. As recent literature points out, energy awareness can be integrated to production scheduling to enable industrial demand side management. Consequently, production loads can be shifted to periods with a lower electricity price for energy cost minimization. However, this may increase the overall production cost, because the labor compensation usually follows the opposite trend with the electricity price. To investigate this trade-off, this paper introduces a scheduling approach that considers both energy consumption and labor shifts. An efficient memetic algorithm is proposed for multi-objective optimization. Numerical experiments on a blow molding process demonstrated two general trade-offs: one between energy cost and labor cost, as well as one between overall production cost and makespan. Therefore, these trade-offs must be considered when performing energy-efficient production scheduling.
\end{abstract}

Keywords: sustainable production scheduling, demand side management, energy model, multi-objective optimization, Pareto analysis

\section{Background}

Industrial energy efficiency (EE) is an indispensable pillar of sustainable production (May et al., 2016). With the penetration of smart grid in manufacturing industry, energy-efficient production scheduling is a promising roadmap toward not only demand-side management (DSM) but also EE. In contrast to conventional scheduling, energy-efficient production scheduling incorporates energy awareness: how much energy consumption and energy cost that a task incurs under volatile electricity prices. Consequently, several aspects need to be embodied beyond the conventional production scheduling. First, onsite power measurement or monitoring should be performed by power meters, sensors, etc. The power data enables energy modeling, which provides empirical energy awareness to the scheduling engine (Gong et al., 2016a). Second, the allowable time slot of a scheduling engine should be sufficiently small to capture the volatile pattern of electricity prices. Among the common electricity pricing schemes in DSM, time-of-use pricing (ToUP) and critical peak pricing change the price within several hours, while real-time pricing (RTP) changes every hour (Gong et al., 2015).

Last but not least, the labor shift should be considered, as the labor cost depends on the shift type (such as early and night shifts) and is sensitive to the load shifting introduced by energy-efficient production scheduling. In fact, energy and labor costs usually follow the opposite trend for the same production task. For instance, in the night and on weekends, the former is lower while the latter is higher. However, the labor shift is 
ignored in energy-efficient production scheduling (Wang and Li, 2014). This trade-off relationship is indicated in (Gong et al., 2017), but is not quantitatively investigated.

The contributions of this paper are twofold. First, an energy-efficient production scheduling problem of general industrial relevance is investigated by integrating labor shifts to account for the trade-off introduced by labor cost. Second, a memetic algorithm (MA) is proposed for effective and efficient multi-objective optimization (MOO).

\section{Problem statement}

The problem is to perform job sequencing and timing of production jobs on a unit process or a bottleneck process of a line, make optimal decision of multiple machine idle modes between adjacent jobs, and assign power consumption states as well as the number and type of human workers, under RTP and without breaking the due date.

Two sets of bi-objectives are defined to reveal the potential trade-offs: minimize energy cost and labor cost (objective 1), and minimize total cost and makespan (objective 2). The total cost in this investigation refers to the joint energy and labor cost, considering the other production cost parts (e.g., material cost) independent of scheduling.

The energy cost includes several subparts: machine startup and shutdown, job processing, machine changeover, as well as machine idling between adjacent jobs. The machine stays off before the first scheduled job and is turned off at the completion of the last job. The labor cost depends on the compensation per labor type and per shift type, as well as the number of each labor type in each shift over time.

All jobs have a release time of zero and the same due date. Each job contains the same type of parts or products, while different jobs have different types of parts or products. A machine changeover is thus required between adjacent jobs. The machine can process only one job at a time, and pre-emption is not allowed.

\section{Labor-integrated energy model}

A state-based energy model is employed to calculate objectives $1 \& 2$ (Sect. 2). It can be implemented by finite state machines (FSMs, or automata) (Gong et al., 2016a), where state retention and event-triggered state transition facilitate calculation over time, and discrete events mimic machine operations (e.g., "power on/off machine" and "start job processing"). This model has threefold benefits. First, it refines energy modeling compared to current studies that assume a constant power for a machine or a few incomplete power states. Second, it reduces the computational burden by focusing on the time points where events occur and ignoring the rest. Third, it facilitates the integration of time-dependant variables such as labor cost. Therefore, in this model, both energy and labor costs are accumulated with state transition.

This energy model employs a multi-scale discrete-time representation. More specifically, it makes the time evolution based on discrete events. It uses three independent time slots (intervals/granules/periods/grids): (1) one for machine power states which is a tuneable parameter (the job duration is included in the general Production state), (2) one for the electricity price which equals the pricing period, and (3) one for labor shifts which is the duration of a shift. The first time slot represents the scheduling granularity, as the latter two are constant inputs. As mentioned in Sect. 1, it should be no larger than the pricing period. While the continuous time representation produces a smaller problem size, the discrete-time representation has several remarkable advantages (Merchan et al., 2016): (1) simpler and flexible for extra features such as variable job release time and due date, complex logistic, linear modeling of inventory, 


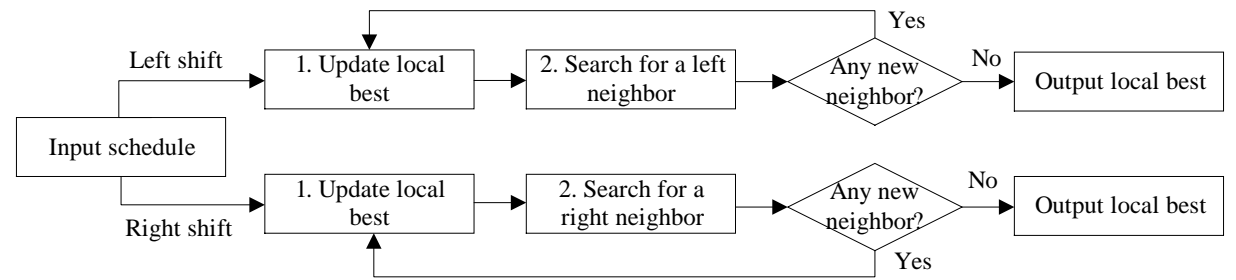

Fig. 1. Hill climbing to hybridize NSGA-II for the memetic algorithm

and other time-varying parameters; (2) more effective not only in reducing computational time, but also in finding better solutions.

\section{Memetic algorithm}

A MA is proposed for MOO of this problem (Sect. 2). It hybridizes the NSGA-II (Deb et al., 2002) with a hill climbing algorithm (Fig. 1). As a renowned multi-objective evolutionary algorithm (MOEA), the NSGA-II has been proven to be good at exploration in a solution space (i.e., global search) within a reasonable time duration. The hill climber is proposed to enhance NSGA-II's exploitation competence (i.e., local search), so that the resulting MA is good at both exploration and exploitation.

This hill climber (Fig. 1) outputs the local best according to an input schedule. A local best is the schedule with the least energy cost and also total cost (calculated by the labor-integrated energy model in Sect. 3). This hill climber considers an input schedule as the local best at the start (step 1). It encounters a new neighbor (step 2) when it shifts only one job of the local best by one electricity pricing period toward the assigned direction and without altering the job sequence of the local best. It then updates the local best (step 1), and iterates this search process (steps 1 \& 2) until it cannot find a new neighbor any more. The search direction can be left (backward in time) or right (forward in time), but must remain fixed in one hill climbing.

Thereby, an input schedule has at most two hill climbing schedules, output by both left and right shifts; and at least one hill climbing schedule, obtained by either left or right shift, or input itself if no better solution is found by left and right hill climbing.

This hill climber applies to every individual in every generation of NSGA-II, without the need of tuning local search parameters (e.g., applied individuals and probability). However, this does not make the search rapidly converge toward a local optimum, as the local best is defined such that some individuals may survive after hill climbing.

\section{Experimental results}

The use case is a Belgian plastic bottle manufacturing plant. As the 17 extrusion blow molding (EBM) lines run in parallel for different types of bottles, our proposed scheduling approach is applicable to any one of these lines.

\subsection{Data and experiment setting}

A line comprises an EBM machine which is the major energy consumer and three downstream auxiliaries including a leakage detector, a weigh checker, and a packaging unit. Three Siemens ${ }^{\circledR}$ PAC 3200 power meters were installed on the major energy subconsumers of an EBM machine: main system, hydraulic system, and extruder.

After over one-year power monitoring, the power profiling identified eight empirical power states: Off, Startup, Idle, Preheat, PreheatIdle, Proheat, ProheatIdle, and 
Production. Besides powering off, three machine idle modes were observed by distinct power profiles, i.e., power level and breakeven duration (necessary time period for switching to and recovering from an idle mode). The collected power data and the empirical state-based energy model can be found in (Gong et al., 2016b).

Three daily shifts are included: early shift (6 AM - 2 PM), late shift (2 PM - 10 PM), and night shift (10 PM - 6 AM). In a night shift, the labor compensation $(€ / h)$ rises by $10 \%$ compared to an early or late shift. For production on weekends, the compensation increases by $36 \%$ in early and late shifts. The two-week RTP date (6 AM 14 to 6 AM 28-Nov-2016) comes from the Belgian electricity spot market. The duration of 10 jobs varies from 2:29:20 to 24:53:21 (h:min:s). The maximum runtime is $2 \mathrm{~h}$.

A design of experiment (DOE) (Montgomery, 2012) was performed with a full factorial setting to tune MA. In this setting, the combination of MA parameter was iterated in potential range of each parameter. Each combination was run for multiple times to achieve statistical significance. Consequently, the optimal configuration (population size, crossover rate, mutation rate) was identified as $(500,0.9,0.3)$.

\subsection{Trade-off analysis}

By setting the objectives $1 \& 2$, respectively, approximations of two Pareto-optimal fronts were obtained, as illustrated in Fig. 2. Both trade-offs are evident: between energy cost and labor cost, as well as between total cost and makespan. The effectiveness of this MA is demonstrated by the approximation front superior to that of the initial population (without MOO). In Fig. 2b, the unique initial non-dominated solution is one of these output by the MA, because a rule-based schedule is included in the population initialization to speed up the convergence of search. This rule is to group all jobs and start production since the beginning of scheduling horizon, such that objective values can all be at a low level. Furthermore, the efficiency of this MA is proved by the approximation front better than that given by NSGA-II in Figs. 2a \& $2 b$. More specifically, when the energy cost is relatively high, it is easier to reduce it (by $26 \%$ ) without compromising much the labor cost (3\% rise) (approximation front of MA, Fig. 2a). However, if the energy cost is already low (by some degree of energy-efficient load shifting), its further decrease has to be compensated by a significant increase in the labor cost (through more night and weekend labor shifts). Moreover, if both cost parts are joint and minimized with makespan, this joint total cost can be decreased by $11 \%$ with little prolongation of makespan (1\%) (approximation front of MA, Fig. 2b).

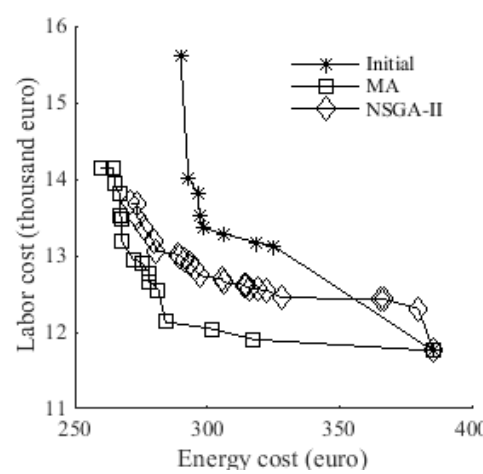

(a)

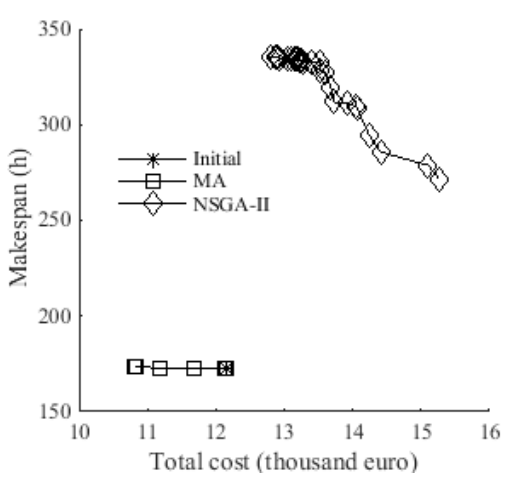

(b)

Fig. 2. Non-dominated solutions for energy cost and labor cost (Fig. 2a), and total cost (energy and labor costs) and makespan (Fig. 2b). The memetic algorithm (MA) achieves the best non-dominated solutions. 
Energy-efficient and labor-aware production scheduling based on multi-objective optimization

Table 1 Multi-objective optimization performance contributed by variable time slots

\begin{tabular}{lllllll}
\hline \multirow{2}{*}{ Time slot } & \multicolumn{2}{c}{$N S$} & \multicolumn{2}{c}{$R N$} & \multicolumn{2}{c}{$T S$} \\
\cline { 2 - 7 } & Objective 1 & Objective 2 & Objective 1 & Objective 2 & Objective 1 & Objective 2 \\
\hline $1 \mathrm{~h}$ & 1 & 14 & 0.11 & 0.21 & n/a & 1.42 \\
$1 \mathrm{~min}$ & 3 & 25 & 0.11 & 0.47 & 1.41 & 0.87 \\
$1 \mathrm{~s}$ & 7 & 16 & 0.78 & 0.32 & 1.01 & 0.59 \\
\hline
\end{tabular}

\subsection{Scalability in number of time slots}

The time slot (the one for machine power states, Sect. 3) was varied to demonstrate the scalability of the proposed scheduling approach. The former configurations remained for fair comparison. Three metrics were used to indicate the MOO performance (Zhang and Chiong, 2016): (1) NS(ts), the number of non-dominated solutions given by time slot $t s$; (2) $R N(t s)$, the degree of Pareto optimality of the non-dominated solutions output by $t s$; (3) $T S(t s)$, the evenness of non-dominated solution distribution of $t s$.

As listed in Table 1, the MOO performance improves when the time slot decreases from $1 \mathrm{~h}$ to $1 \mathrm{~s}$, although the amount of time slots increases by four orders of magnitude (from 336 to $1,209,600$ ). This not only demonstrates the scalability of the MA, but also reveals that a finer time slot can effectively enhance the MOO performance.

However, one exception exists in Table 1: $R N$ under objective 2 drops from 0.47 to 0.32 when the time slot shrinks from $1 \mathrm{~min}$ to $1 \mathrm{~s}$. This is due to the search in the solution space where one of the major tasks is to look for an optimal or nearly-optimal job timing. A finer search step covers all the solutions with a coarser step (given that they are in multiplier relationship). Nevertheless, an exhaustive iteration of the finer solution space cannot be guaranteed within a limited runtime budget, although the search efficiency of this MA is already enhanced beyond NSGA-II. As nearly $70 \%$ of the global non-dominated solutions come from the time slot larger than $1 \mathrm{~s}(R N(1 h)=0.21$, $R N(1 \mathrm{~min})=0.47$, Table 1$)$, a further MOO enhance strategy is to run the MA under different time slots and merge the non-dominated solutions in the end. A compromise of this strategy would be deterioration in TS, given the TS trend in Table 1.

\subsection{Discussion}

Despite the exception found in Sect. 5.3, a fine scheduling time slot is obliged for effective and efficient MOO, since a coarse time slot will lose many good scheduling solutions (Table 1). This highlights the contribution of high-precision energy modeling and energy-efficient production scheduling, compared to the literature where both energy modeling and production scheduling usually have coarse timing granularity.

The MA is a representative of evolutionary algorithms (EAs). EAs are independent of scheduling problems, although problem-specific heuristics or measures may be introduced for performance enhancement. They provide approximate solutions (which are usually good or at least reasonable) in reasonable time for various large and complex scheduling problems which are conventionally intractable.

Comparatively, mathematical programming has a prevalent computational challenge. In (Hadera et al., 2016), the number of binary variables and constraints increases with the number of time slots, such that the problem size is only limited to several time slots. The formulation in (Merchan et al., 2016) is complex and poor in scalability, depending on the model, method, and objective. The CPU time of 100,000 sec in (Lappas and Gounaris, 2016) is very long while some problem instances can still not be solved. 
However, EAs cannot guarantee real optimality. Unlike in mathematical programming, real optimality is unknown in EAs. It is hard to measure how far the approximate solutions of EAs are exactly from the real optimality. Besides, the optimization efficiency of EAs highly depends on parameter tuning.

Overall, EAs are a practical optimization technique for production scheduling, since a shop floor requires to quickly get a good schedule even for complex and large problems.

\section{Conclusions}

The existing energy-efficient production scheduling research omits the increasing labor cost due to load shifting toward lower electricity pricing periods (usually nights and weekends). To fill this gap, this paper proposes an energy-efficient and labor-aware scheduling approach.

In this approach, a state-based and event-triggered energy model uses a multi-scale discrete time representation and integrates labor shifts for simple but effective calculation of the objective values: energy cost, labor cost, and makespan. Moreover, a memetic algorithm (MA) is proposed by hybridizing NSGA-II with hill climbing.

In a plastic bottle manufacturing case, two trade-offs of general significance were revealed: between energy cost and labor cost, as well as between a sum of these two cost parts and makespan. Besides, this MA's effectiveness and efficiency are proved by its superior approximation of Pareto-optimal front compared to that of the initial population and NSGA-II, respectively. This MA's timing scalability is also proved. The future work is to extend this scheduling approach to a larger shop floor scale.

\section{References}

K. Deb, A. Pratap, S. Agarwal, T. Meyarivan, 2002, A fast and elitist multiobjective genetic algorithm: NSGA-II, IEEE Trans. Evol. Comput. 6, 182-197.

X. Gong, T. De Pessemier, W. Joseph, L. Martens, 2015, An energy-cost-aware scheduling methodology for sustainable manufacturing, Procedia CIRP, 29, 185-190.

X. Gong, T. De Pessemier, W. Joseph, L. Martens, 2016a, A generic method for energy-efficient and energy-cost-effective production at the unit process level, J. Clean. Prod., 113, 508-522. X. Gong, T. De Pessemier, W. Joseph, L. Martens, 2016b, A power data driven energy-costaware production scheduling method for sustainable manufacturing at the unit process level, IEEE 21st Int. Conf. Emerging Technologies and Factory Automation, Berlin, Germany, pp. 1-8. X. Gong, M. Van der Wee, T. De Pessemier, S. Verbrugge, D. Colle, L. Martens, W. Joseph, 2017, Energy- and labor-aware production scheduling for sustainable manufacturing: a case study on plastic bottle manufacturing, Procedia CIRP, 61, 387-392.

H. Hadera, R. Labrik, J. Mäntysaari, G. Sand, I. Harjunkoski, S. Engell, 2016, Integration of energy-cost optimization and production scheduling using multiparametric programming, Comput. Aided Chem. Eng., 38, 559-564.

N.H. Lappas, C.E. Gounaris, 2016, Comparison of continuous-time models for adjustable robust optimization in process scheduling under uncertainty, Comput. Aided Chem. Eng., 38, 391-396. G. May, B. Stahl, M. Taisch, 2016, Energy management in manufacturing: Toward eco-factories of the future - A focus group study, Appl. Energ. 164, 628-638.

A.F. Merchan, H. Lee, C.T. Maravelias, 2016, Discrete-time MIP methods for production scheduling in multistage facilities, Comput. Aided Chem. Eng., 38, 362-367.

D.C. Montgomery, 2012, Design and Analysis of Experiments, 8 ed. John Wiley \& Sons. Y. Wang, L. Li, 2014, Time-of-use based electricity cost of manufacturing systems: Modeling and monotonicity analysis, Int. J. Prod. Econ., 156, 246-259.

R. Zhang, R. Chiong, 2016, Solving the energy-efficient job shop scheduling problem: a multiobjective genetic algorithm with enhanced local search for minimizing the total weighted tardiness and total energy consumption, J. Clean. Prod., 112(4), 3361-3375. 